$63^{\text {ème }}$ Congrès de la SFCO, 02029 (2015)

DOI:10.1051/sfco/20156302029

(C) Owned by the authors, published by EDP Sciences, 2015

\title{
COMMUNICATION
}

\section{Du nouveau en pathologie salivaire : le carcinome sécrétoire pseudomammaire}

\author{
Jouan $\mathrm{R}^{* *}$, Lupu Bratiloveanu $\mathrm{P}^{*}$, Le Bescond $\mathrm{Y}^{*}$ \\ * Centre Hospitalier de Roanne, Chirurgie maxillo-faciale et Stomatologie, 42300 Roanne \\ ** Hospices Civils de Lyon, 69000 Lyon
}

Mots Clés : Glandes salivaires, Carcinome sécrétoire pseudomammaire, ETV6-NTRK3.

Introduction. Depuis 2010, une nouvelle forme de carcinome des glandes salivaires a été identifiée : le Carcinome sécrétoire pseudomammaire (ou MASC pour mammary analogue secretory carcinoma). Cette entité tumorale présente de nombreuses ressemblances avec le carcinome sécrétoire du sein, en particulier une translocation t(12;15)(p13;q25) intéressant les gènes ETV6 et NTRK3. Cette tumeur maligne mal connue et de présentation non spécifique risque de voir sa prévalence augmentée par les progrès du diagnostic génétique.

Observation. Le cas d'un patient de 42 est rapporté ici, montrant l'aspect aussi peu spécifique que possible de cette tumeur maligne. Sa prise en charge diagnostique, chirurgicale et post-chirurgicale sera présentée. Discussion. Par le faible nombre de cas publiés à ce jour (moins de 100), cette pathologie est mal connue. Elle a probablement longtemps été confondue avec le carcinome à cellules acineuses. Sa prise en charge n'est pas standardisée et de nombreux diagnostics sont posés a posteriori. Il conviendra sans doute à l'avenir de définir un arbre décisionnel afin d'éviter une prise charge empirique.

Nom et adresse du conférencier

\section{Robin JOUAN}

Hospices Civils de Lyon

69000 Lyon (France)

robin.jouan@chu-lyon.fr

This is an Open Access article distributed under the terms of the Creative Commons Attribution License 4.0, which permits unrestricted use, distribution, and reproduction in any medium, provided the original work is properly cited. 